Please do not remove this page

RMIT

UNIVERSITY

\title{
Width dependence of inherent TM-mode lateral leakage loss in silicon-on-insulator ridge waveguides
}

Webster, Mark; Pafcheck, Robert; Koch, Thomas; Mitchell, Arnan

https://researchrepository.rmit.edu.au/esploro/outputs/9921863279501341/filesAndLinks?institution=61RMIT_INST\&index=null

Webster, M., Pafcheck, R., Koch, T., \& Mitchell, A. (2007). Width dependence of inherent TM-mode lateral leakage loss in silicon-on-insulator ridge waveguides. IEEE Photonics Technology Letters, 19(6), 429-431. https://doi.org/10.1109//pt.2007.891979

Published Version: https://doi.org/10.1109//pt.2007.891979

Repository homepage: https://researchrepository.rmit.edu.au

(C) 2007 IEEE

Downloaded On 2023/04/26 23:11:30 +1000

Please do not remove this page 


\title{
Width Dependence of Inherent TM-Mode Lateral Leakage Loss in Silicon-On-Insulator Ridge Waveguides
}

\author{
M. A. Webster, Member, IEEE, R. M. Pafchek, A. Mitchell, Member, IEEE, and T. L. Koch, Fellow, IEEE
}

\begin{abstract}
We report the first experimental observation in the optical domain of a dramatic width-dependent lateral leakage loss behavior for the TM-like mode of tight vertical confinement ridge waveguides formed in silicon-on-insulator. The lateral leakage loss displays a series of sharp cyclic minima at precise waveguide widths, and appears to be inherent to waveguide geometries of central importance to a wide variety of active devices in silicon photonics requiring lateral electrical access. This behavior is not predicted by the often-used effective-index-based methods, but is understood phenomenologically and also compared to prior numerical analysis and predictions of leaky mode behavior. It is shown that TM-like mode operation, critical to the operation of some active component designs, will require precision control of waveguide dimensions to achieve high performance.
\end{abstract}

Index Terms-Leaky waves, optical losses, optical waveguides, silicon-on-insulator (SOI) technology.

\section{INTRODUCTION}

$\mathbf{I}$ $\mathrm{N}$ THE rapidly advancing area of silicon photonics, the large refractive-index-contrast of the silicon-on-insulator (SOI) material system allows for very tight vertical field confinement and thus enables greater performance for a wide variety of important active devices such as modulators and promising source configurations. The ridge waveguide geometry (inset in Fig. 1) is often required in SOI photonics for lateral electrical access to the optical mode region in such active devices. It is not generally appreciated that the high refractive-index-contrast and common active device dimensions of these tight vertical confinement ridge waveguides can make the TM-like mode inherently leaky in the lateral direction for many waveguide geometries of great interest.

Here, we present experimental observations in the optical domain of the lateral leakage loss behavior for the TM-like mode. The leakage loss displays a dramatic width dependence with a series of sharp cyclic minima at very precise waveguide widths

Manuscript received Nov. 27, 2006. This work was supported by National Science Foundation Grant ECS-0335067, by Pennsylvania BFTDA Grant ME\#21-116- 0014, by the Army Research Laboratory Cooperative Agreement W911NF-04-2-0015, and by the Bandwidth Foundry Pty Ltd., Australia.

M. A. Webster, R. M. Pafchek, and T. L. Koch are with the Center for Optical Technologies, Lehigh University, Bethlehem, PA 18015 USA (e-mail: tlkoch@ lehigh.edu).

A. Mitchell is with the School of Electrical and Computer Engineering, RMIT University, Melbourne, VIC 3001, Australia.

Color versions of one or more of the figures in this letter are available online at http://ieeexplore.ieee.org.

Digital Object Identifier 10.1109/LPT.2007.891979

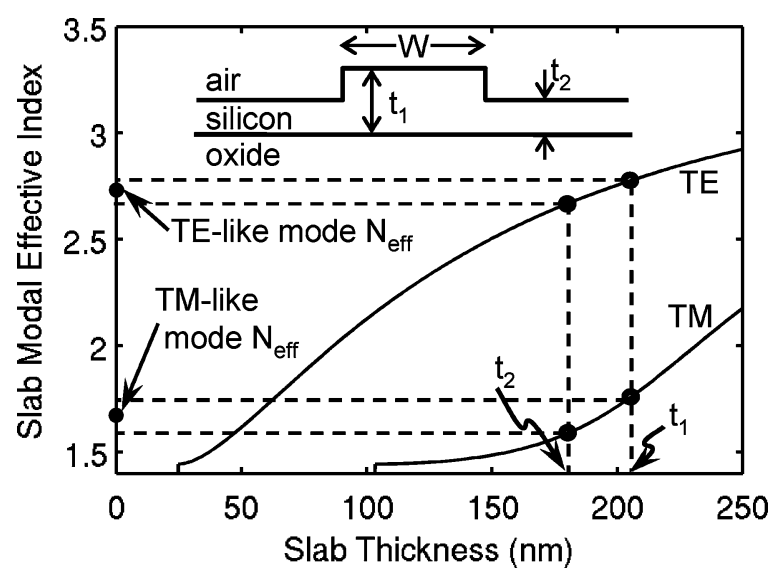

Fig. 1. Slab mode indexes used in effective index calculations. The modal indexes for the guided TE-like and TM-like modes, respectively, lie intermediate between their slab values for slab thicknesses $t_{1}$ and $t_{2}$. We use index values of $n_{\mathrm{Si}}=3.475$ and $n_{\mathrm{SiO} 2}=1.444$ at $1.55-\mu \mathrm{m}$ wavelength.

[1]. A simple phenomenological model is presented and also compared to prior numerical analysis and predictions of leaky mode behavior.

\section{THEORY OF LATERAL-LEAKAGE LOSS}

Under an equivalent slab model for the ridge waveguide geometry (inset in Fig. 1), the lateral leakage loss for the TM-like mode is due to TM/TE mode conversion at the ridge boundary [2]-[4]. It should be emphasized that this effect is not caused by any surface or side-wall roughness. With reference to slab waveguide dispersion curves in Fig. 1, for the case of TE-like modes, this mode conversion at the boundary cannot lead to any propagating field or leakage loss in the lateral cladding since longitudinal phase-matching requires that any fields generated at the ridge boundaries will be laterally evanescent in the slab lateral cladding for both the TE and TM generated fields. However, in the case of the TM-like mode, while phase-matching requires that the lateral TM slab mode be evanescent in the lateral cladding, any TE slab field component generated at the boundary is phase-matched to a laterally propagating TE slab mode at some angle $\theta$ in the lateral slab cladding region.

This phase-matching is illustrated in Fig. 2(a). Here $\beta_{\mathrm{TE}}$ and $\beta_{\mathrm{TM}}$ represent the propagation constants of the TE-like and TM-like modes of the ridge waveguide, respectively. This diagram illustrates that the propagation constant of the TM-like mode is much less than that of the TE-like mode, and for many 


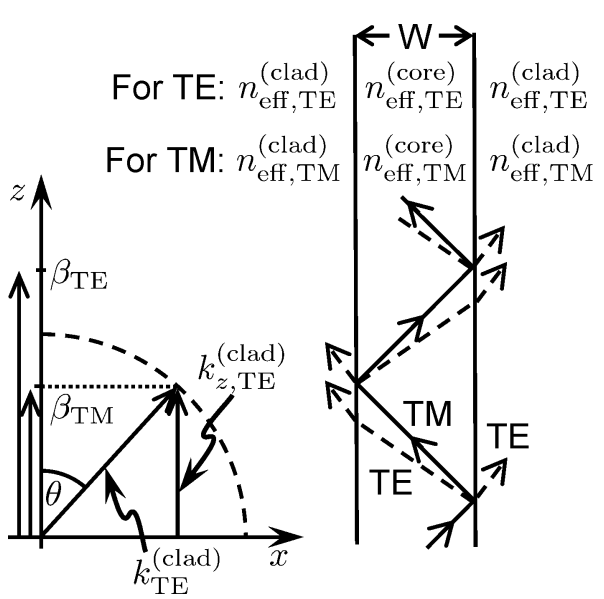

Fig. 2. Phase-matching diagram showing TM-like waveguide mode phase-matched to a propagating TE slab mode in the lateral cladding.

practical rib guides may also lie below the propagation constant of the unguided TE slab mode $k_{\mathrm{TE}}^{\text {(clad) }}$. Since this slab mode is unguided by the rib, it may propagate at any angle and if $\beta_{\mathrm{TM}}<k_{\mathrm{TE}}^{(\mathrm{clad})}$, it is possible to rotate $k_{\mathrm{TE}}^{(\mathrm{clad})}$ by an angle $\theta$ such that the TE-slab and TM-guided mode are phase-matched in the $z$ direction. Since the guided mode is then phase-matched to a radiation mode, it is possible that leakage may occur if there is some means for mode conversion.

For a thin enough lateral slab thickness $t_{2}$, and certainly in the case of a strip or "wire" waveguide where $t_{2}=0$, the TE slab effective index can lie below the TM-like mode effective index, and hence, avoid this phase-matched leakage. However, for electrical access, these designs may not be practical, and thus, this leakage loss must be well understood.

The leakage process is illustrated in Fig. 2(b). Since the $z$ components of all propagation constants are conserved, all waves develop the same relative phase along the length of guide and we can discuss phase in the lateral direction only.

Starting at the bottom, a TM mode is guided by the rib and is represented as the solid ray incident on the right wall, where the angle of incidence is such that TM total internal reflection occurs. However, due to the step discontinuity at the rib wall, mode conversion from TM to TE can occur, and it can be shown from mode-matching calculations [4] that TE transmitted and reflected propagating waves are produced that are approximately equal in magnitude, but are $\pi$ radians out of phase. The reflected TE radiation mode traverses across the core as shown by a dashed line. Upon total internal reflection, the TM mode experiences a negative phase shift $\phi_{\mathrm{TIR}}$ and the combination of $\phi_{\mathrm{TIR}}$ with the phase from a single traverse of the guide to the left is zero for the fundamental mode.

At the left rib wall, the TM mode generates additional small reflected and transmitted TE propagating waves. The new transmitted TE wave, with a relative phase of $\pi$ radians as noted above, combines with the previous reflected TE wave that has traversed the guide with a phase shift of $k_{x, \text { TE }}^{(\text {core }} W$. Thus, if this phase shift across a single traverse for the TE in the core is a multiple of $2 \pi$, the TE waves will interfere destructively. This

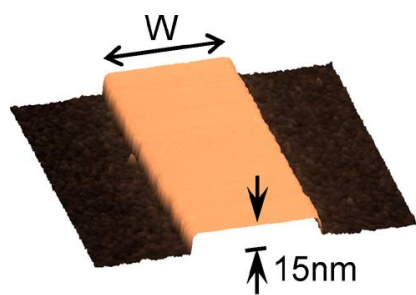

(a)

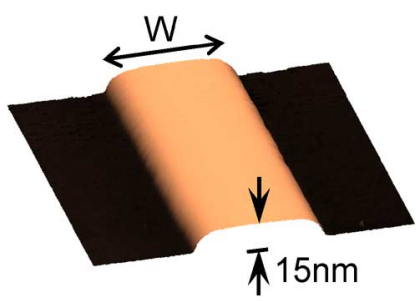

(b)
Fig. 3. AFM images of waveguide profiles formed by (a) wet-etching and (b) thermal oxidation.

leads to a width dependence for the leakage minima that satisfies a resonance-like condition [3] of $k_{x, \mathrm{TE}}^{(\mathrm{core})} W=m \cdot 2 \pi$, or alternatively stated,

$$
W=\frac{m \cdot \lambda}{\sqrt{\left(n_{\mathrm{eff}, \mathrm{TE}}^{\text {(core })}\right)^{2}-N_{\mathrm{eff}, \mathrm{TM}}^{2}}}, \quad m=1,2,3 \ldots
$$

Here, $N_{\text {eff, TM }}$ has a weak $W$ dependence, so some care must be used if precision is required. From this argument, we would expect to see significant leakage loss for TM propagation, except at precise, specific waveguide widths satisfying the resonance condition (1), where the leakage loss would be greatly reduced due to destructive interference of radiating TE waves. It is also interesting to note that the next higher order mode will be lossy at these widths, since the radiated fields add in phase for this mode. This may allow relatively wide guides with effectively single-mode behavior.

\section{EXPERIMENTAL RESULTS}

We fabricated a series of ridge waveguides with widths varying from 0.5 to $1.8 \mu \mathrm{m}$ in 50-nm increments with thickness $t_{1}=205 \mathrm{~nm}$ and $t_{2}=190 \mathrm{~nm}$ (i.e., a 15-nm ridge height) on an SOI wafer with a $2-\mu \mathrm{m}$ buried oxide layer thickness. These waveguides were formed by wet-etching, and as previously measured at a wavelength of $1.55 \mu \mathrm{m}$ for the TE-like mode, have low propagation losses of about $0.7 \mathrm{~dB} / \mathrm{cm}$ [5].

We also fabricated waveguides using a thermal oxidation process that results in smoother and rounded sidewalls. Fig. 3(a) and (b) shows atomic force microscope (AFM) profiles of the waveguide surfaces.

The total relative transmitted fiber-coupled power was measured as a function of waveguide width for both the TE and TM input polarizations and is presented in Fig. 4. Here each data point corresponds to the measured power averaged over ten nominally identical waveguides with a length of $1.5 \mathrm{~cm}$. It can be readily observed that the TE-like mode has a net loss weakly dependent upon waveguide width, whereas the TM-like mode has large loss except at precise values of width.

A simple effective index model was used to calculate the TE and TM modal effective indices. These were then substituted into (1) to obtain predicted widths of 0.72 and $1.44 \mu \mathrm{m}$ for the first two resonances which are in excellent agreement with the measured results, as shown in Fig. 4.

The data of Fig. 4(b) show that the waveguides with a smooth rounded sidewall still display the TM-like mode lateral leakage 

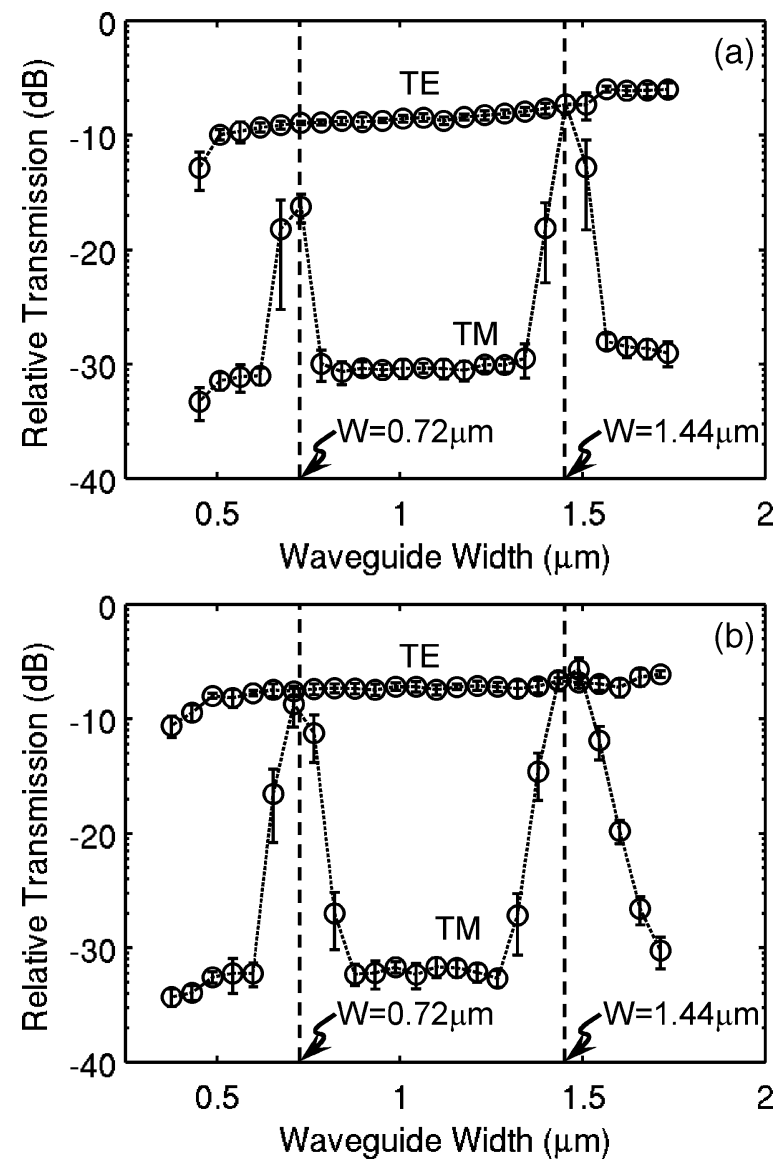

Fig. 4. Experimentally measured transmission losses (log scale) for the fundamental TE-like and TM-like modes for SOI waveguides formed by (a) wetetching and (b) thermal oxidation. Note dramatic width dependence of TM-like mode loss, and sharp reduction in losses at very precise values of waveguide width, in excellent agreement with predictions of (1).

loss, but show a broader width dependence at the loss minima. This is believed to be due to a weaker TM/TE conversion at the ridge boundaries.

\section{DISCUSSION}

The characteristics presented in Fig. 4 exhibit some subtle features that warrant further discussion. First, it should be noted that the resonant leakage cancellation is not perfect. This is particularly evident for the first peak of Fig. 4(a). This imperfect cancellation was predicted [4] and can be explained by the fact that near cutoff, close to the critical angle, the magnitudes of the transmitted and reflected TE waves produced by an incident TM wave are not exactly equal. This imbalance reduces as the angle approaches glancing, and thus, we see improved resonant cancellation for stronger guiding with the wider rib. It should also be noted that the leakage loss in general reduces with increasing rib width. This was also predicted [3], [4] and can be explained to the reduced mode conversion from TM to TE as the incident angle approaches $90^{\circ}$ (where the conversion drops to zero).

Also of significant interest is the reduced leakage behavior exhibited by the oxidized samples in Fig. 4(b). Here the narrow rib exhibits almost the same loss as the TE guided mode and the wider rib appears to possibly provide even lower propagation loss in the TM mode. The resonances are also significantly broader than the rectangular etched ribs. This suggests that engineering of the leakage behavior to broaden the range of low-loss widths may be possible. Optimal designs and effective simulation strategies for investigating these designs are currently under investigation.

This demonstration of design requirements to realize low-loss TM mode guidance will have significant consequences for the practicality of TM-based devices, including slot waveguide with a very thin horizontal low-index layer that provide large slotlayer confinement factor [6], [7]. Successful engineering of TM guides that are both low-loss and fabrication tolerant will be an important technology for this branch of Si photonics.

\section{CONCLUSION}

We have presented the first experimental observation of unusual cyclic leaky mode behavior for the TM-like mode in the optical domain, and in an SOI waveguide. This compares well with theoretical predictions. Experimental observations of nonrectangular geometries suggest that engineering for improved TM leakage properties may be possible.

\section{REFERENCES}

[1] M. A. Webster, R. M. Pafchek, A. Mitchell, and T. L. Koch, "Experimental observation of precision widths mitigating inherent leakage in silicon-on-insulator ridge waveguides," in IEEE LEOS Annual Meeting, Montreal, Canada, Oct. 2006, Postdeadline paper PD 1.5.

[2] S. T. Peng and A. A. Oliner, "Guidance and leakage properties of a class of open dielectric waveguides: Part I-Mathematical formulations," IEEE Trans. Microw. Theory Tech., vol. 29, no. 9, pp. 843-855, Sep. 1981.

[3] A. A. Oliner, S. T. Peng, T. I. Hsu, and A. Sanchez, "Guidance and leakage properties of a class of open dielectric waveguides: Part II-New physical effects," IEEE Trans. Microw. Theory Tech., vol. 29, no. 9, pp. 855-869, Sep. 1981.

[4] K. Ogusu, "Optical strip waveguide: a detailed analysis including leaky modes," J. Opt. Soc. Amer., vol. 73, no. 3, pp. 353-357, 1983.

[5] M. A. Webster, R. M. Pafchek, G. Sukumaran, and T. L. Koch, "Low-loss quasi-planar ridge waveguides formed on thin silicon-on-insulator," Appl. Phys. Lett., vol. 87, p. 231108, 2005.

[6] V. R. Almeida, Q. Xu, C. A. Barrios, and M. Lipson, "Guiding and confining light in void nanostructure," Opt. Lett., vol. 29, no. 11, p. 1209,2004

[7] N. N. Feng, J. Michel, and L. C. Kimerling, "Optical field concentration in low-index waveguides," IEEE J. Quantum Electron., vol. 42, no. 9 , pp. 883-888, Sep. 2006. 